\title{
UNA RELACIÓN OLVIDADA: HEIDEGGER Y SCHELER ${ }^{1}$
}

\author{
Enrique V. Muñoz Pérez ${ }^{2}$ \\ Universidad Católica del Maule \\ enmunoz@ucm.cl
}

\begin{abstract}
Resumen
Este artículo pretende mostrar el lugar que ocupa el pensamiento de Max Scheler en la reflexión de Martin Heidegger. Para ello, presenta, por un lado, las principales etapas de dicha relación desde ambas perspectivas filosóficas y, por otro lado, propone que la crítica a la teoría del conocimiento de características neokantianas es la influencia más determinante del pensamiento de Scheler en el proyecto filosófico de Heidegger.
\end{abstract}

Palabras Clave: metafísica, antropología, amor (Liebe), angustia (Angst), neokantianismo.

\section{Abstract}

We seek to show the role of Max Scheler's thought in the philosophy of Martin Heidegger. We present, on the one hand, the main phases of their relationship from the philosophical perspectives of both, and, on the other hand, we submit that Scheler's critique of neo-Kantian theory of knowledge was the chief influence exercised by Scheler on Heidegger's philosophical project.

KEYWORDS: metaphysics, anthropology, love, anguish, Neokantianism.

\section{Introducción}

$\overline{R A}$ La relación entre Martin Heidegger (1889-1976) y Max Scheler (1874-1928) es bien conocida a partir de las referencias que hace el mismo Heidegger en

1 Este trabajo fue escrito en el marco del Proyecto de Investigación Postdoctoral sobre el tema "Una relación olvidada: La influencia del pensamiento filosófico de Max Scheler en la concepción ontológica del hombre de Martin Heidegger" (2007-2009) con apoyo financiero del Fondecyt (Chile) (Proyecto $\mathrm{N}^{\circ}$ 3085016). Agradezco a mi colega profesor Dr. César Lambert O., por la revisión de mis traducciones.

2 El autor es Profesor de Filosofía y Licenciado en Filosofía por la Pontificia Universidad Católica de Chile y Doctor en Filosofía por la Albert-Ludwigs Universität Freiburg (Alemania), con una tesis denominada Der Mensch im Zentrum, aber nicht als Mensch. Zur Konzeption des Menschen in der ontologischen Perspektive Martin Heideggers, bajo la dirección de Günter Figal. 
distintos momentos de su obra sobre Scheler, particularmente en el así llamado “entorno" (Umkreis) de Ser y Tiempo (1927)3 ${ }^{3}$. En esta época es posible reconocer algunos comentarios positivos del pensamiento de Scheler por parte de Heidegger. Piénsese, por ejemplo, en pasajes de Kant y el problema de la metafísica (1929) o en la lección magistral de Marburgo Metaphysische Anfangsgründe der Logik im Ausgang von Leibniz (semestre de verano 1928). Sin embargo, menos estudiada, y al mismo tiempo menos conocida, es la influencia que tuvo el pensamiento de Scheler sobre el de Heidegger. La intención de este artículo es, por consiguiente, presentar algunas características y alcances de esta desatendida relación, en especial, en el mencionado período de la reflexión heideggeriana. En otras palabras, la pregunta que me interesa dilucidar es: ¿hasta qué punto influye Max Scheler en el proyecto filosófico de Heidegger, que es posible identificar particularmente en el entorno de $\mathrm{Ser}$ y Tiempo?

De este modo, dividiré el presente trabajo en los siguientes momentos: (1) De Heidegger a Scheler; (2) De Scheler a Heidegger, y (3) Observación final.

\section{(1) De Heidegger a Scheler}

En la relación Heidegger-Scheler hay, a lo menos, tres momentos que son claramente distinguibles ${ }^{4}$ : 1) un juicio riguroso y crítico de Heidegger contra Scheler; 2) una aproximación gradual entre Heidegger y Scheler, y 3) finalmente, la identificación de algunos puntos de convergencia filosófica entre ambos pensadores. Ilustraré, en lo que sigue, cada una de estas etapas de la mencionada relación.

1.1. Como ejemplo del juicio negativo contra Scheler, podemos mencionar, entre otros, pasajes de Fenomenología de la vida religiosa (semestre de invierno 1920/21 - semestre de verano 1921) o de Ontología (Hermenéutica de la facticidad) - semestre de verano 1923).

Max Scheler es uno de los principales responsables de la recuperación del pensamiento de San Agustín en Alemania, según sostiene Kisiel en su conocida obra The genesis of Heidegger's Being and Time (1995, p. 192). En este contexto, se entiende la referencia negativa y peyorativa que hace Heidegger sobre Scheler al inicio de la lección magistral Agustín y el neoplatonismo (incluida, en español, en el volumen Estudios sobre mística medieval). La interpretación scheleriana de San Agustín es, a juicio de Heidegger, "sólo una recepción secundaria de este círculo

3 Se entiende normalmente por "entorno" de Ser y Tiempo el período que va aproximadamente entre 1924 y 1930 (Vigo 2003, p. 143).

$4 \quad$ Sigo aquí la tesis de Michael Großheim expuesta en el artículo Heidegger und die Philosophische Anthropologie (Großheim, 2003, pp. 333 s.). 
de ideas, aderezado con fenomenología" (Heidegger 1997b, p. 24; cursiva mía). La expresión "círculo de ideas" apunta a la influencia histórico-espiritual de San Agustín, la que, según Heidegger, es identificable desde la fundamentación de la teología medieval hasta la moderna escuela apologética católica francesa, pasando incluso por Lutero. El análisis scheleriano del pensamiento de San Agustín, que es también fenomenológico, se presentaría para Heidegger como insuficiente.

Por otro lado, Heidegger sostiene en Ontología (Hermenéutica de la facticidad), citando textos de Scheler, que él

"se mueve, a la manera tradicional, entre cuestiones cuyo planteamiento se ha quedado anticuado y resulta artificial; sólo gracias a la depurada manera fenomenológica de ver y explicar es tanto más funesto. Scheler pretende determinar el "lugar metafísico...dentro del todo del ser, del mundo y de Dios", el "género homo". Pretende deshacerse "del atavío mítico-simbólico" de la idea y apoderarse de la cosa misma. [...]"

"Lo que el hombre sea" - ¡sentido, miras, hermenéutica de esta cuestión! El hombre es "la intención y el gesto de la "trascendencia" misma", el que busca a Dios, "un "entre", [.] "límite"”. (Animal-Dios, ambos asumidos), "un eterno "hacia afuera"”, la "puerta de entrada" de la gracia, "... la única idea de "hombre" con sentido [es] por completo un "teo-morfismo", la idea de un X que es una copia viva y finita de Dios, un semejante a él, - ¡una de sus infinitas siluetas recortadas sobre la gran pared del ser! Claramente: ipuro decorado!, ;literatura, ficción!" (Heidegger 1999, p. 44 s. Cursiva mía).

Heidegger critica, por un lado, la interpretación que hace Scheler del método fenomenológico: Scheler pretende asir "la cosa misma", en este caso, el hombre. Sin embargo, su procedimiento filosófico se ve teñido de la metafísica tradicional. En otras palabras, la confusión de Scheler se encuentra en que su antropología filosófica, al estar fundamentada en la metafísica tradicional, no hace diferencia entre los distintos modos de ser, e iguala en su tratamiento a las plantas, a los animales y al hombre mismo. Por otro lado, la concepción scheleriana del hombre peca de "teomorfismo", es decir, está determinada por perspectivas teológicas o religiosas. De ahí el juicio perentorio de Heidegger: la concepción scheleriana del hombre es un intento superficial e inútil.

1.2. Con todo, es posible constatar más tarde un cambio significativo en la interpretación heideggeriana sobre el pensamiento filosófico de Scheler, en el sentido de un reconocimiento parcial y limitado de la antropología filosófica scheleriana. Ello acontece en Ser y Tiempo (1927), pero también en Prolegomena zur Geschichte des Zeitbegriffs (1925).

En el caso de Ser y Tiempo, la referencia positiva que hace Heidegger acerca de Scheler se lleva a cabo en el marco de una descripción del tema del hombre en la filosofía contemporánea ( $(10)$. Los hitos de este movimiento son, entre otros, Dilthey, Bergson, Husserl y Scheler mismo. Ninguno de ellos, sostiene Heidegger, se preguntó por el ser de la persona. Sin embargo, la reflexión scheleriana tiene un mérito: sus estudios concluyeron que 
"la persona no debe ser pensada jamás como una cosa o como una substancia; ella "es más bien la unidad concomitante e inmediatamente vivida del vivir de las vivencias- y no una cosa solamente pensada detrás y fuera de lo inmediatamente vivido". La persona no es un ser substancial y cósico. Tampoco puede agotarse el ser de la persona en ser sujeto de actos racionales regidos por determinadas leyes".

"La persona no es ni cosa, ni substancia, ni objeto" (Heidegger 1997a, p. $72 \mathrm{~s}$. Cursiva mía).

En este pasaje, Heidegger cita, sin decirlo explícitamente, El formalismo en la ética y la teoría material de los valores de Max Scheler ${ }^{5}$. Heidegger refiere aquí al "anuario" (Jahrbuch) de Husserl. Y este dato no es menor, porque en dicha publicación apareció no solo Ética scheleriana en el año 1913, sino que también una obra muy significativa para mi interpretación, como mostraré más adelante: Zur Phänomenologie und Theorie der Sympathiegefühle und von Liebe und Haß.

Volviendo al texto de Heidegger, él considera que si bien la intuición de Scheler es correcta, esto es, que el hombre (la persona) no se encuentra al mismo nivel ontológico que un objeto, una cosa, una planta o un animal; ella está sustentada en la ontología del "estar-ahí" (Vorhandenheit) y no responde a una pregunta central: “CCómo determinar ontológicamente de una manera positiva el modo de ser de la persona?" (Heidegger 1997a, p. 73. Cursiva mía). Esta búsqueda de un nuevo fundamento ontológico para pensar lo humano marca el tratamiento que hace Heidegger de dicha temática en gran parte de su obra. El hombre, dirá más tarde Heidegger, tiene que sacar a la luz el Dasein que se encuentra en él ${ }^{6}$.

Por otro lado, en la lección magistral Prolegomena zur Geschichte des Zeitbegriffs (1925), Heidegger expresa también una opinión ponderada sobre el trabajo de Scheler, aunque sin dejar de deslizar críticas a su antropología filosófica. Lo que llama la atención de la siguiente cita es que Heidegger comenta, positivamente, el mismo texto que unos pocos años antes era calificado de "puro decorado" o de "literatura".

5 "Como indicará lo que sigue, hay en estas definiciones una cosa muy exacta, a saber: que nunca puede ser pensada la persona como una cosa o una sustancia que tuviere cualesquiera virtud o fuerzas" (Scheler 1942, p. 11. Cursiva mía) Cf. también Scheler 1976, p. 82.

6 Estoy pensando, por ejemplo, en el giro "Dasein en el hombre" (Dasein im Menschen) que emerge en Kant y el problema de la metafísica (1929). "Si el hombre sólo es hombre a raíz del Dasein en él, la pregunta por lo que es más primordial que el hombre no puede ser, en principio, una pregunta antropológica. Toda antropología, aun la filosófica, supone ya al hombre como hombre. El problema de la fundamentación de la metafísica se enraíza en la pregunta por el Dasein en el hombre, es decir, en la pregunta por su fundamento íntimo, por la comprensión del ser como finitud esencialmente existente" (Heidegger 1996, p. 193. Cursiva mía). 
"La única idea del hombre (Scheler) llena de sentido es enteramente un teomorfismo, la idea de una $\mathrm{X}$, la que es una reproducción finita y viva de Dios, una comparación de una de sus infinitas siluetas recortadas sobre la gran pared del ser. Ello es formulado, empero, más con un tinte literario que científicamente bien pensado; con todo, presenta la determinación de Scheler del ser del hombre" (Heidegger 1994, p. 181. Cursiva mía) ${ }^{7}$.

Heidegger reconoce ahora el intento scheleriano de buscar el ser del hombre. Y ello no es un hecho aislado. En el mismo texto es posible identificar una relativa confluencia entre la perspectiva de Heidegger y la posición de Scheler, por ejemplo, a propósito de las reflexiones de Scheler acerca del Dasein (en su sentido original de "existencia") y del mundo; del saber como una "relación de ser" (Seinsverhältnis) o acerca del tiempo ${ }^{8}$.

1.3. Curiosamente, unos pocos años después, Heidegger expresa claramente su admiración y su acuerdo en algunos puntos con Scheler. La admiración se expresa tanto en la dedicatoria que hace Heidegger a Scheler de su libro Kant y el problema de la metafísica (1929), como en el detallado recuerdo que hace Heidegger de Scheler en su lección magistral sobre Leibniz, Metaphysische Anfangsgründe der Logik im Ausgang von Leibniz (1928), inmediatamente acontecido su deceso. Las principales coincidencias entre su trabajo y el de Scheler son precisamente comentadas en la mencionada lección:

"En nuestro último diálogo prolongado en diciembre de 1927 concordamos en cuatro puntos: 1. El problema de la relación sujeto-objeto tiene que ser planteado de un modo enteramente nuevo, esto es, libre de los intentos hechos hasta ahora. 2. No se trata de una pregunta de la así llamada teoría del conocimiento; esto es, dicho problema primeramente no ha de ser planteado en consideración de un sujeto que capta un objeto; este aprehender no puede ser puesto a la base al modo del punto de partida. 3. Tiene un significado central para la posibilidad de la metafísica y se halla en la más interna relación con su problema fundamental. 4. Lo más esencial: Este es el momento, precisamente por la desolación de la situación pública filosófica, para atreverse nuevamente a dar el paso hacia la verdadera metafísica, esto es, desarrollarla desde de su fundamento" (Heidegger 1990, p. 165) .

7 "Die einzig sinnvolle Idee von Mensch (Scheler) ist ganz und gar ein Theomorphismus, die Idee eines X, das endliches und lebendiges Abbild Gottes ist, ein Gleichnis seiner, einer seiner unendlichen Schattenfiguren auf der Wand des Seins. Das ist allerdings mehr literatenhaft formuliert als wissenschaftlich durchdacht, aber zeigt doch Schelers Bestimmung des Seins des Menschen" (Heidegger 1994, p. 181).

8 Cf. Heidegger 1994, p. 302 s.

9 "In unserem letzten längeren Gespräch im Dezember 1927 sind wir uns über ein Vierfaches einig geworden: 1. Das Problem der Subjekt-Objekt-Beziehung ist vollkommen neu 
En esta larga cita, Heidegger se concentra en el tratamiento de dos temas, a mi juicio, centrales en la época inmediatamente posterior a la aparición de Ser y Tiempo: la relación sujeto-objeto y su concepción coyuntural de metafísica. El primer caso, es quizás una de las principales coincidencias entre el pensamiento de Heidegger y el de Scheler: la relación sujeto-objeto no puede ser entendida como una problemática que se inserte exclusivamente en la denominada teoría del conocimiento, sino que debe ser reformulada. En otras palabras, Heidegger y Scheler coinciden en la idea de que el hombre "tiene mundo" o que "está en el mundo", por lo que no se lo puede separar arbitrariamente de su entorno. La otra gran temática es el surgimiento a fines de los años 20 en el pensamiento de Heidegger de la "metafísica del Dasein" (Metaphysik des Daseins), temática que se enmarca en la profundización, desarrollo y/o reformulación de la ontología fundamental presentada en Ser y Tiempo.

Precisamente es esta "metafísica del Dasein" la protagonista de otro de los textos en que Heidegger comenta detalladamente la reflexión scheleriana: su conferencia inédita llamada Philosophische Anthropologie und Metaphysik des Daseins ${ }^{10}$. En este texto, Heidegger enjuicia positivamente el trabajo filosófico de Scheler; califica la reflexión scheleriana como modélica, porque Scheler intentó esclarecer la íntima relación entre la Antropología filosófica y la Metafísica. Antropología filosófica y Metafísica fueron los polos en que su preguntar y su pensar se desenvolvieron. Así lo dice Heidegger:

"Pero precisamente cuando nosotros nos dirigimos hacia el centro del problema fundamental, se requiere de una determinada orientación preparatoria. A saber, no partir de tendencias que son muchas veces confusas y que se mantienen la mayor parte en los márgenes de la filosofía, sino que a partir del presente de un filosofar, que una en sí ambas tendencias en un potente querer y en una amplia plenitud y movimiento: el trabajo filosófico de Max Scheler (Antropología filosófica y Metafísica en general fueron los dos polos, alrededor de los cuales giraron su preguntar y pensar siempre más clara y apasionadamente)" (Heidegger, Inédito, p. 5) ${ }^{11}$.

zu stellen, und zwar frei von den Versuchen, die bisher aufgetreten sind. 2. Es ist keine Frage der sogenannten Erkenntnistheorie; d.h., es ist nicht primär zu stellen mit Rücksicht auf ein Subjekt, das ein Objekt erfaßt; dieses Erfassen darf im Ansatz nicht zugrunde gelegt werden. 3. Es hat zentrale Bedeutung für die Möglichkeit der Metaphysik und steht im innersten Bezug zu ihrem Grundproblem. 4. Das Wesentlichste: der Augenblick ist da, gerade bei der Trostlosigkeit der öffentlichen philosophischen Lage, den Überschritt in die eigentliche Metaphysik wieder zu wagen, d.h., sie von Grund aus zu entwickeln" (Heidegger, 1990, p.165)

10 Esta conferencia fue leída por Heidegger el 24 de enero de 1929 en la Sociedad Kant (Kant-Gesellschaft) en Frankfurt y aparecerá en el volumen 80, titulado Vorträge, de las obras completas de Martin Heidegger.

11 "Aber gerade wenn wir ins Zentrum des Grundproblems streben, bedarf es einer bestimmten vorbereitenden Anweisung. Zwar nicht ausgehen von den mannigfaltigen, oft 
Uno puede sostener, entonces, que para Heidegger es plausible la intuición de Scheler de vincular la Antropología filosófica y la Metafísica. Sin embargo, Heidegger hace presente, al mismo tiempo, una importante diferencia con Scheler: el significado de la expresión "Metafísica". La metafísica, para Scheler, principalmente en la época final de su pensamiento, está fundada en una base teológica o religiosa. Por su lado, Heidegger desarrolla, como ya he sostenido, en esa época la "metafísica del Dasein". Esta puede ser caracterizada brevemente del siguiente modo: Heidegger sostiene en la conferencia Philosophische Anthropologie und Metaphysik des Daseins que la metafísica del Dasein debe "aclarar la posibilidad interna de la comprensión del ser a partir de la esencia del Dasein" y a partir de ello "dar el lineamiento para el tipo de formulación del problema fundamental de la metafísica y con eso predeterminar la problemática de la metafísica en el todo" (Heidegger, Inédito, p. 24). ${ }^{12}$ Dado que éste es el interés de Heidegger, es comprensible que considere que la concepción metafísica de Scheler no es lo suficientemente radical: ella no se cuestiona el sentido, el derecho y la necesidad de que la pregunta por la esencia del hombre sea el centro del filosofar. Para Heidegger, hablar de "antropología filosófica" es únicamente posible sobre la base de la ontología fundamental o de sus variaciones.

He mostrado hasta el momento los vaivenes de la relación Heidegger-Scheler, desde la perspectiva del filósofo de Meßkirch. Presentaré, a continuación, el punto de vista del pensador de Munich.

\section{(2) De Scheler a Heidegger}

La manera en que alude Scheler a Heidegger puede ser dividida en dos grandes estadios: 1) Algunas alusiones ocasionales que desarrolla Scheler sobre Heidegger en distintos momentos de su obra, y 2) la reseña que realiza Max Scheler sobre Ser y Tiempo. Comentaré, a continuación, ambos puntos.

2.1. En un texto denominado Die deutsche Philosophie der Gegenwart (1922), Max Scheler pretende retratar "la más grande de las épocas espirituales de Alemania"

verworrenen und meist nur ganz am Rande des Philosophierens sich haltenden Tendenzen, sondern von der Gegenwart eines Philosophierens, das beide Fragerichtungen in einem starken Wollen und weiten Fülle und Beweglichkeit in sich vereinigte: der philosophischen Arbeit Max Schelers (Philosophische Anthropologie und Metaphysik überhaupt waren die beiden Polen, um die sein Fragen und Denken immer eindeutiger und leidenschaftlicher kreiste)" (M. Heidegger, Inédito, p. 5).

12 "Die Metaphysik des Daseins muß die innere Möglichkeit des Seinsverständnisses aus dem Wesen des Daseins aufhellen und damit die Vorzeichnung geben für die Art der Ausarbeitung des Grundproblems der Metaphysik und damit vorausbestimmen die Problematik der Metaphysik im Ganzen" (Heidegger, Inédito, p. 24). 
(Scheler 2005, p. 338), que contrasta con la caótica situación política en que se encuentra el país, a pocos años de concluir la Primera Guerra Mundial. La fenomenología es el paradigma de este renacer filosófico alemán, la que en un tiempo relativamente corto ha desarrollado una gran influencia, que no es comparable con ninguna otra escuela de pensamiento. En ese marco, Scheler le dedica algunas líneas al trabajo de Heidegger, previo a Ser y Tiempo: Scheler lo destaca como un joven representante del movimiento fenomenológico, "proveniente de la escuela de Rickert." (Scheler 2005, p. 329); pero todavía más, lo considera junto con él como un nuevo aporte a la reflexión filosófica. Scheler dice del trabajo de Heidegger que "aquí existe un nuevo tipo de fenomenología y líneas fundamentales de un sistema" (Scheler 2005, p. 330) ${ }^{13}$ Entre esas líneas, Scheler destaca las siguientes:

\section{Heidegger}

1. El giro ontológico. 2. El giro de la filosofía de la vida. 3. El giro histórico. 4. El giro individual. 5. El giro pragmático relativo. 6. El giro antropológico. 7. El giro concreto. 8. Fenomenología Hermenéutica. 9. Ruptura con el cartesianismo. 10. Rechazo de la filosofía de la razón occidental (desde los griegos). 10. Influencia: Kierkegaard, Protestantismos, Scheler, Jaspers, Dilthey, Graf Yorck (Scheler 2005, p. 330. Cursiva mía) ${ }^{14}$.

Llama la atención cómo Scheler se ve a sí mismo como una influencia en el pensamiento de Heidegger.

2.2. Uno de los hitos más desconocidos de la relación Scheler-Heidegger es la recensión que éste realiza sobre Ser y Tiempo ${ }^{15}$. El escrito se denomina Aus kleineren Manuskripten zu Sein und Zeit (1927). En la mencionada reseña, Scheler critica el énfasis de Heidegger en términos como "angustia", "muerte" o "cuidado" y los contrasta con la noción de "amor" (Eros). Además, intenta identificar la tesis principal de la obra de Heidegger. Así lo dice Scheler:

13 “Anders mit Heidegger: Hier liegt ein neuer Typus von Phänomenologie vor und Grundlinien eines Systems" (Scheler 2005, p. 330).

14 "Heidegger 1. Die ontologische Wendung. 2. Die lebensphilosophische Wendung. 3. Die historische Wendung. 4. Die individuelle Wendung. 5. Die relativ pragmatische Wendung. 6. Die anthropologische Wendung. 7. Die konkrete Wendung. 8.Hermeneutische Phänomenologie. 9. Bruch mit Cartesianismus. 10. Ablehnung der abendländischen Vernunftphilosophie (Seit Griechen). 11. Einfluß : Kierkegaard, Protestantismos, Scheler, Jaspers, Dilthey, Graf Yorck" (Scheler 2005, p. 330).

15 Entre los escasos trabajos en español sobre la relación Heidegger-Scheler, cabe mencionar los artículos de Raúl Gabás Pallás (2002) y de Jesús Adrián Escudero (2007). Una nota sobre Scheler, particularmente sobre el tópico de "El saber y la cultura", es desarrollada en un reciente libro por Jorge E. Rivera (2007). 
"El hombre es el ser que "se ocupa" de sí mismo y que desde la angustia se abre a "sí mismo" y a la muerte, como si no se ocupara de sí mismo, sino del "útil" (bienes, mercancías, trabajo,-cuidado). ¡Esta es la tesis de Heidegger! Pero no es así: ¡Ciertamente el hombre se ocupa de sí mismo, pero sólo en solidaridad con las cosas y su fundamento!

El primer giro hacia lo inmanente mundano es efectivamente Eros, no el rechazo, la angustia, la huida de sí" (Scheler 1976, p. 294) ${ }^{16}$.

Posteriormente, Scheler enfatiza su preferencia por el "Eros", en comparación con el concepto heideggeriano de "angustia", en su relación con el mundo. Lo que nos hace accesible el mundo es "el "amor", no la angustia. La angustia presupone la esfera mundana ya abierta (Pérdida del miedo del animal y de los primitivos)" (Scheler 1976, p. 294) ${ }^{17}$. También Scheler discute la interpretación heideggeriana de la muerte. Ella no es percibida correctamente. "La muerte llega a ser más revestida con la vida y sus progresos y es al mismo tiempo el punto mediante el cual comienza la creciente autonomía del espíritu. La muerte es "cosecha", no auto-aniquilamiento" (Scheler 1976, p. 294) ${ }^{18}$. En otras palabras, Scheler considera que hay algo más que la mera finitud y que la muerte no es el límite último de la existencia humana.

Por otro lado, Scheler piensa que la filosofía heideggeriana ostenta un marcado sesgo teológico protestante, en cuyo fondo resuena un tipo de neocalvinismo, representado por la Teología de Karl Barth. Scheler toma distancia de este rasgo de la obra de Heidegger, porque el modo en que esta teología pregunta por la verdad no es propiamente filosófico, sino que histórico y "esta particular Teología del Dios absoluto trascendente se presenta como una enseñanza ilimitada, histérica y superada" $\left(\right.$ Scheler 1976, p. 295) ${ }^{19}$.

Más adelante, Scheler comenta el conocido concepto heideggeriano de "Dasein" y su relación con la noción de "ser humano" (Mensch):

16 "Der Mensch ist das Sein, "dem es um sich geht" und das aus Angst "sich" und dem Tode so "tut", als ginge es ihm nicht um sich selbst, sondern um das "Zeug" (Besitz, Ware, Arbeit, - Sorge). Das ist Heideggers These! Aber so ist es nicht: Es geht dem Menschen wohl um sich - aber nur solidarisch mit den Dingen und ihrem Grund!

Die erste Wendung zum Welt-immanenten ist doch Eros, nicht Abstossung, Angst, Flucht vor sich" (Scheler 1976, p. 294).

17 "Das, was uns die Welt aufschliesst, ist "Liebe" nicht Angst. Die Angst setzt die erschlossene Weltsphäre voraus (Angstlosigkeit des Tieres und der Primitiven)" (Scheler 1976, p. 294).

18 "Der Tod wird anziehender mit dem Leben und seinem Fortschreiten und ist zugleich der Punkt, da sich die wachsende Selbständigkeit des Geistes abhebt. Der Tod ist "Ernte", nicht Selbst-vernichtung" (Scheler 1976, p. 294).

19 “(...) und da mir ausserdem diese besondere Theologie der absoluten Gott-transzendenz als eine masslose hysterische übersteigerte Lehre vorkommt“" (Scheler 1976, p. 295). 
"Heidegger posee ni el más mínimo medio para dividir la esencia del ocasional Dasein, del ser humano. El Dasein es -Heidegger en su total y ocasional concreción- con color de pelo y cualquier forma de su cuerpo. La tesis de que "el hombre es sólo un modo de ser" sobrepasa la esencia del hombre tanto como ella limita al ocasional Heidegger. Los modos de ser se juzgan desde la esencia". (Scheler 1976, p. 296) ${ }^{20}$.

La crítica de Scheler a Heidegger, a propósito de uno de los conceptos centrales de la reflexión heideggeriana, no es menor: el Dasein no puede ser escindido del ser humano, ni puede ser dejada de lado su corporalidad. Todavía más, la existencia como modo de ser propio del ser humano no puede sino que ser entendida a partir de la esencia humana. En otras palabras, a juicio de Scheler, Heidegger pierde de vista que el ser del hombre es ser persona.

Con todo, Scheler recomienda fervorosamente la obra de Heidegger: su libro Ser y Tiempo es una de las obras más originales, más libres e independientes de las tradiciones filosóficas. Más aún, Scheler alaba en Heidegger "la arremetida radical y, a pesar de ello, estrictamente científica de los más hondos problemas de la filosofía" (Scheler 1976, p. 304) ${ }^{21}$. En otras palabras, Ser y Tiempo es para Scheler una pequeña revolución del pensamiento filosófico alemán de su época.

\section{(3) Observación final}

Más allá de las etapas que puedan identificarse en la relación entre Heidegger y Scheler y, a su vez, de los comentarios de Scheler sobre Heidegger, la mutua relación entre Scheler y Heidegger es un hecho. Ello es confirmado también por el editor de las obras completas de Scheler en alemán, M.S. Frings, quien sostiene que Scheler ocupa un lugar destacado junto a Husserl y Heidegger en la filosofía contemporánea. Además, enfatiza Frings, ellos se conocieron entre sí, se influyeron mutuamente y produjeron sus obras principales entre los años 1900 y $1927^{22}$.

20 "Heidegger besitzt keinerlei Mittel, zu scheiden das Wesen vom zufälligen Dasein des Menschen. Das Dasein ist -Heidegger in seiner ganzen zufälligen Konkretion- mit Haarfarbe und jeder zufälligen Gestalt seines Körpers. Die These der "Mensch ist nur Seinsweise" übersteigt das Wesen des Menschen so sehr, als sie der zufällige Heidegger untersteigt, Die Seinsweise richtet sich nach dem Wesen" (Scheler 1976, p. 296).

$21 \quad$ “(...) ein radikales und doch streng wissenschaftliches Angreifen der höchsten Probleme der Philosophie" (Scheler 1976, p. 304).

22 "There can be no doubt that Scheler does have this intermediary place between Husserl und Heidegger from a historical viewpoint of contemporary philosophy, although Husserl, Scheler, and Heidegger knew each other personally and produced many of their influential works roughly in the same period of time (1900-1927). Anyone familiar with Husserl's phenomenology will easily see Scheler's few attachments to Husserl as well 
Con todo, me interesa abordar el siguiente punto, a modo de síntesis final: ¿cuál es la influencia más determinante del pensamiento de Max Scheler en el proyecto filosófico de Martin Heidegger? Me parece que, sin duda alguna, la crítica a la teoría del conocimiento de características neokantianas.

Mi sospecha se ve confirmada cuando uno tiene presente los comentarios de Hans Blumenberg, quien en una reciente publicación póstuma sostiene que Scheler, junto con publicar El formalismo en la ética y la teoría material de los valores en el año 1913, publicó otra obra ese mismo año llamada Zur Phänomenologie und Theorie der Sympathiegefühle und von Liebe und Haß, en cuyo apéndice denominado "Über den Grund zur Annahme der Existenz des fremden Ich" trata sobre el problema de la intersubjetividad. El punto que, empero, subraya Blumenberg es que en esta obra, Scheler estableció, por primera vez, que la relación entre ontología y antropología no podía sustentarse más sobre un realismo inspirado en la teoría del conocimiento. ${ }^{23}$ En otras palabras, Scheler intenta ya en el año 1913 buscar una nueva fundamentación para la relación entre ontología y antropología, decisión que, como sostuve anteriormente, influye positiva y decisivamente en Heidegger. El mejor ejemplo de ello, lo constituye su conferencia inédita Philosophische Anthropologie und Metaphysik des Daseins.

\section{Referencias bibliográficas}

Blumenberg, H. (2006), Beschreibung des Menschen. Frankfurt a.M.: Editorial Suhrkamp.

Escudero, Jesús Adrián (2007), "Hacia una fenomenología de los afectos”, Thémata. 39: 365-368.

Figal, G. (1999), Martin Heidegger zur Einführung. HYamburgo: Editorial Junius.

Frings, M. S, (1997), The Mind of Max Scheler. Milwaukee-Wisconsin: Marquette University Press.

Gabás Pallás, Raúl (2002), "La fenomenología de los sentimientos en Max Scheler y el concepto de afección en Martin Heidegger", Enrahonar, Quaderns de Filosofia. 34: 31-46.

as Scheler`s many desviations from him as to phenomenological procedures. However, it requires an intense study as a special subject, of Scheler's own works to establisch him as a forerunner of the Philosophy of Dasein. There exist beyond any question passages in Scheler's writings that, although the term Existenz (Dasein) is not used, remind one of the existential atmosphere that started to arise in Germany during the twenties" (Frings 1997, p. 3).

23 "In diesem Werk Schelers war zum ersten Mal die Verbindung von Ontologie und Anthropologie als ein nicht mehr erkenntnistheoretisch ansetzender 'Realismus' hergestellt" (Blumenberg 2006, p. 22). 
Großheim, M. (2003), "Heidegger und die Philosophische Anthropologie", HeideggerHandbuch (Leben-Werk-Wirkung), editado por Dieter Thomä. StuttgartWeimar: Editorial J.B. Metzler, pp.333-337.

Heidegger, M. (1990), Metaphysische Anfangsgründe der Logik, GA Bd. 26, editado por Klaus Held. Frankfurt a.M.: Editorial Vittorio Klostermann..

(1994), Prolegomena zur Geschichte des Zeitbegriffs, GA Bd. 20, editado por Petra Jaeger. Frankfurt a.M.: Editorial Vittorio Klostermann.

(1996), Kant y el problema de la metafísica, traducido por Gred Ibscher Roth. México: Editorial Fondo de Cultura Económica.

(1997a), Ser y Tiempo, traducido por Jorge Eduardo Rivera. SAntiago de Chile: Editorial Universitaria.

(1997b), Estudios sobre mística medieval, traducido por Jacobo Muñoz. Madrid: Ediciones Siruela.

(1999), Ontología. Hermenéutica de la facticidad, traducido por Jaime Aspiunza. Madrid: Alianza Editorial.

(2006), Introducción a la fenomenología de la religión, traducido por Jorge Uscatescu. México: Ediciones Siruela-Fondo de Cultura Económica.

(Inédito), "Philosophische Anthropologie und Metaphysik des Daseins", Vorträge ( que será editado por Bernd Heimbüchel, en la GA Bd. 80).

Kisiel. T. (1995), The genesis of Heidegger's Being \& Time. Berkeley, Los Angeles, London: University of California Press, p. 192.

Lambert, C. (2002), Philosophie und Welt beim jungen Heidegger. Frankfurt a.M.: Editorial Peter Lang.

Rivera, Jorge Eduardo (2007), En torno al ser. Santiago de Chile: Bricklediciones.

Scheler, M. (1942), "Ética. Nuevo ensayo de fundamentación de un personalismo ético", traducido por Hilario Rodríguez S. Revista de Occidente, Madrid, 1942.

(1976), Späte Schriften, GW Bd. 9, editado por Manfred S. Frings. Berna: Editorial Francke.

(2005), Wesen und Formen der Sympathie/Die deutsche Philosophie der Gegenwart, GW Bd. 7, editado por Manfred S. Frings. Bonn: Editorial Bouvier.

Vigo, A. (2003), "Verdad, libertad y trascendencia en Heidegger. La radicalización de un motivo central de Sein und Zeit en los escritos de los años 1929-1930", La filosofía como pasión. Homenaje a Jorge Eduardo Rivera Cruchaga en su $75^{\circ}$ cumpleaños, editado por Patricio Brickle. Madrid: Editorial Trotta, pp. 143-190. 